\title{
Soziologie der Gewalt
}

\author{
Andreas Braun
}

Online publiziert: 14. Juli 2020

(C) Der/die Autor(en) 2020, korrigierte Publikation 2021

Hoebel, Thomas, und Wolfgang Knöbl: Gewalt erklären! Plädoyer für eine entdeckende Prozesssoziologie. Hamburg: Hamburger Edition 2019. 223 Seiten. ISBN: 978-3-86854-335-3. Preis: $€ 22$.

Die in den letzten Dekaden der (noch jungen) sozialwissenschaftlichen Gewaltforschung geführten Debatten um konzeptuelle Weiterentwicklungen haben zu fruchtbaren Erkenntnissen geführt. Mit Blick auf das zentrale Anliegen jeglicher wissenschaftlichen Forschung, das Erklären, lässt sich aber ein deutliches Manko konstatieren: Es wurde grundlegend, versäumt zur Frage der Erklärung klar Stellung zu nehmen“" (S. 8), so Thomas Hoebel und Wolfgang Knöbl zu Beginn ihrer lesenswerten Monografie.

Der vernachlässigte Blick auf methodologische Grundfragen bezüglich explikativ relevanter Kausalannahmen, ontologischer wie epistemologischer Prämissen oder der konträren Behandlung von Beschreibung und Erklärung (S. 14-18, S. 49, S. 133-144) untergräbt den Autoren zufolge nicht nur das (künftige) Potenzial gewaltsoziologischer Forschung, sondern verweist auf eine drohende Stagnation (S. 195) - vor allem im Zuge situationistischer Forschungen. Damit konfrontiert „Gewalt erklären!" die aktuelle gewaltsoziologische Forschung in doppelter Hinsicht: Zum einen soll und muss jenes „wissenschaftstheoretische Desinteresse“ (S. 18) aufgehoben und Fragen nach methodologischen Grundlagen sowie der Erklärung für künftige Weiterentwicklungen ernstgenommen werden - zu Recht. Zum anderen sinnen die Autoren mit ihrem „Plädoyer für eine entdeckende Prozesssoziologie“ auf

\footnotetext{
A. Braun $(\square)$

Fakultät für Soziologie, Universität Bielefeld

Universitätsstr. 25, 33615 Bielefeld, Deutschland

E-Mail: andreas.braun@uni-bielefeld.de
} 
die Überwindung der drohenden Stagnation mithilfe eines prozessualen Ansatzes, der gegenüber anderen in der Lage ist, Gewalt valide zu erklären (S. 15).

Beide Aspekte sind hierbei argumentativ stringent verbunden und werden vor dem Hintergrund der eigenen wissenschaftstheoretischen Prämissen entfaltet (Kapitel 2). In der Sichtung der Forschungslandschaft als Ökologie werden - unter Rekurs auf A. Abbott und J.L. Martin - systematisch drei Heuristiken rekonstruiert, die unter „den Schlagworten ,Motive“, ,Situationen“ und ,Konstellationen““ (S. 38) subsumiert werden. Im Zuge eines weiten Erklärungsverständnisses, das Erklären und Beschreiben nicht konträr behandelt, und einem Kausalitätsverständnis, das sich konsequent auf Ereignistransitivitäten, Temporalitäten und maßgeblich auf (dynamische) Verursachungen statt auf Ursachen bezieht (Kapitel 2, S. 48-60, S. 157), wird jenen Heuristiken ein kausaltheoretisch wie explikativ defizitärer Charakter attestiert (Kapitel 3). Dieser wird bezüglich der konstatierten Aporien nicht zuletzt auf das Problem der „Mikro-Makro-Semantik“ (S. 144) zurückgeführt (Kapitel 4). So sind z. B. raum-zeitlich-stabile Motivannahmen (S. Kalyvas) eben nicht ex ante handlungsbestimmend, sondern nur retrospektiv und situationsabhängig reflexiv rekonstruierbar (S. 62-80). Situationsheuristiken sind ohne Bestimmung des Situation-Motiv-Verhältnisses und der Beachtung „makrokontextueller Randbedingungen“ (S. 100) ebenso defizitär, was u. a. auf einem unklaren Situationsbegriff, der Ablehnung transitiver Motive (R. Collins) und dem Problem (selbst-)inszenierungsabhängiger situativer Wahlmöglichkeiten (L.A. Fujii) beruht (S. 80-100). Selbst auf (makro-)strukturellen Gewaltmärkten/-räumen (G. Elwert, T. Snyder, J. Baberowski) oder generalisierenden Verhaltensregeln und Freiheitsgraden organisationaler Mitglieder (S. Kühl) basierende Konstellationsheuristiken ignorieren z. B. nichtintendierte Konsequenzen sowie situative Bedingungen, prozessuale Ereignisverkettungen und kausale Temporalitäten (S. 104-123).

In der Konsequenz ihres Plädoyers verdeutlichen die Autoren letztlich (Kapitel 4), dass auch eine zirkuläre (Mikro-Makro-)Verbindung keine Lösung verspricht (S. 124f.): Infolge einer generell unklaren ,Unterscheidung von Epistemologie und Ontologie“ (S. 130) führt die „Mikro-Makro-Semantik“ (S. 144) in eine ,theoretische und konzeptionelle Sackgasse“ (S. 129) und ,unvermeidlich zu dubiosen Kausalkonstruktionen“ (S. 144). Was mit Blick auf die hierfür herangezogenen tauschtheoretischen (P. Blau) und Ebenen des Sozialen differenzierenden (u.a. N. Luhmann) Ansätze überzeugend dargelegt wird, muss bezüglich des Modells soziologischer Erklärung hingegen kritisch betrachtet werden. Die explikative Plausibilität des Grundmodells (H. Esser) mag wegen einer ,wahlweise reduktionistisch oder emergentistisch“ (S. 134) angelegten Mikro-Makro-Verbindung zwar äußerst fraglich sein (S. $133 \mathrm{f}$.), ohne eine detaillierte Auseinandersetzung mit den Weiterentwicklungen mehrstufiger (auch prozesserklärender) Modelle kann dieser Ablehnung aber nicht ohne Weiteres zugestimmt werden.

Gleichwohl entspringt es einer nachvollziehbaren forschungsprogrammatischen Logik, demgegenüber den prozessualen Ansatz (Kapitel 5) ins Feld zu führen. Mit Blick auf die (erstens) zentrale Bedeutung ,,[k]ausale[r] Ereignistransitivität[en]“ (S. 159) lassen sich explikativ sensibilisierend sequenzielle Kausalketten genau rekonstruieren und somit detaillierte Einblicke in Gewaltdynamiken gewinnen (S. 159-166), was bezüglich antizipierender Deutungs-, Bewertungs- und Wahr- 
nehmungsmuster lokal anwesender wie abwesender Personen (zweitens) durch übersituational geltende (generalisierte) „Prämissen des Handelns und Erlebens“ (S. 167) gestützt wird (S. 167-173). Hierzu gehört zudem (drittens) die Indexikalität von Gewalt und (viertens) die Historizität von Individuen. Hiermit geraten „situierte [...] und kontextgebundene“ (S. 173) Handlungsspezifika in den Blick u. a. durch sinnbestimmende Aspekte (z.B. konkrete Handlungsorte und externe Bedingungen) und zeitlich spezifisch relationale individuelle (Beziehungs-)Verstrickungen (S. $173 \mathrm{ff}$.). Mittels einer relationalen Ontologie bezüglich der Sozial- und Zeitdimension (S. 176f.), methodisch angelehnt an die Ethnografie und mit detaillierten sequenziellen Ereignisrekonstruktionen arbeitend (S. 176-200), ermöglicht dies schließlich, ,intentionale, situative und konstellationsbezogene Argumente zu verknüpfen“ (S. 145f.). Empirisch an den Massenerschießungen in Józefów und den Anschlägen auf Charlie Hebdo verdeutlicht (S. 158-200), ist Gewalt somit als prozessuales Ergebnis von Situationen transzendierenden handlungsrelevanten Eintrittsentscheidungen und Mitgestaltungsprozessen in soziokulturell variablen Kollektiven zu fassen, die in den situativ verketteten Sequenzen transitiv präsent sind und zugleich relational durch die Menge (vergangener, gegenwärtiger und künftig) erfahrener wie erlebter (extra-)lokaler Deutungs-, Bewertungs- und Beziehungsmuster bestimmt werden.

Ohne Frage, die vier sensibilisierenden Konzepte geben Forschenden bezüglich gewaltsoziologischer Weiterentwicklungen ein instruktives methodologisches Instrumentarium an die Hand und haben für die Verknüpfung von Situationen, Konstellationen und Intentionen explikatives Potenzial. Jenseits der organisational gerahmten Fallbeispiele, bleibt gegenwärtig aber offen, inwieweit dies konkret auf andere Gewaltereignisse übertragen werden kann - eine Verursachung durch organisationale Eintrittsentscheidungen und Mitgestaltungsprozesse liegt, z. B. bei Amoktaten oder häuslicher Gewalt, nicht klar auf der Hand. Gleichwohl können solche Fälle über die vorgeschlagene sequenzielle Ereignisrekonstruktion sicher fruchtbar rekonstruiert werden; auch wenn hier der prozesssoziologisch nicht unumstrittene begrifflichdefinitorische Status von Ereignissen, die als zentrale und ,kleinste zeitliche Untersuchungseinheit“ (S. 189) fungieren, noch unklar ist und ohne „Stoppregeln“ offen bleibt, wann die ,genaue Rekonstruktion der Ereignisverkettung“ (S. 166) erreicht ist.

Dennoch ist der Aufruf des Gewalterklärens ernst zu nehmen und das Plädoyer für prozessuale Gewalterklärungen auch für künftige Diskussionen und Weiterentwicklungen empfehlenswert und instruktiv. Ob es sich hierbei aber um ,den entscheidenden Ansatzpunkt“ (S. 157) zur Überwindung der ,derzeit drohende[n] Stagnation in der soziologischen Gewaltforschung“ (S. 195) handelt, bleibt abzuwarten. Da „das heuristische Potenzial einer Methodologie prozessualen Erklärens für die Gewaltforschung [...] bei Weitem noch nicht ausgeschöpft ist“" (S. 198), würde sich gerade mit Blick auf die prozessual dynamischen zeitlichen Verursachungen und den (methodologischen) Stellenwert der vier sensibilisierenden Konzepte künftig noch deren Klärung als notwendige und/oder hinreichende Bedingungen - im Anschluss an die Verweise auf C. Ragin - anbieten.

Funding Funding Open Access funding enabled and organized by Projekt DEAL. 
Open Access Dieser Artikel wird unter der Creative Commons Namensnennung 4.0 International Lizenz veröffentlicht, welche die Nutzung, Vervielfältigung, Bearbeitung, Verbreitung und Wiedergabe in jeglichem Medium und Format erlaubt, sofern Sie den/die ursprünglichen Autor(en) und die Quelle ordnungsgemäß nennen, einen Link zur Creative Commons Lizenz beifügen und angeben, ob Änderungen vorgenommen wurden.

Die in diesem Artikel enthaltenen Bilder und sonstiges Drittmaterial unterliegen ebenfalls der genannten Creative Commons Lizenz, sofern sich aus der Abbildungslegende nichts anderes ergibt. Sofern das betreffende Material nicht unter der genannten Creative Commons Lizenz steht und die betreffende Handlung nicht nach gesetzlichen Vorschriften erlaubt ist, ist für die oben aufgeführten Weiterverwendungen des Materials die Einwilligung des jeweiligen Rechteinhabers einzuholen.

Weitere Details zur Lizenz entnehmen Sie bitte der Lizenzinformation auf http://creativecommons.org/ licenses/by/4.0/deed.de.

Andreas Braun Dr., Mitarbeiter in den Arbeitsbereichen Soziologische Theorie und Organisationssoziologie an der Fakultät für Soziologie der Universität Bielefeld. Forschungsschwerpunkte: Soziologische Theorien, Handlungstheorien, Gewaltsoziologie, Neurosoziologie. Aktuelle Publikationen: Gewaltsoziologie im Wandel. Über Situationen, Prozesse und Ordnungen als Bestandteile zur Erforschung von Gewalt (im Erscheinen). Bestandsaufnahme soziologischer Gewaltforschung. Wiesbaden 2020 (hrsg. mit T. Kron, im Erscheinen); Interpersonal Violence as an Intrinsic Part oft he Civilizing Process. A Neurosociological Approach. European Journal of Sociology 60, 2019; Zur Entdeckung verkörperter Gewalt. Zeitschrift für Theoretische Soziologie 8, 2019. 